\title{
Lung Macrophages Accumulate Novel Cytosolic Inclusions With Vaping: A Case Report From an Asymptomatic Habitual Vaper
}

DA Armstrong ( $\square$ David.A.Armstrong@hitchcock.org )

Dartmouth-Hitchcock Medical Center https://orcid.org/0000-0003-1748-2520

\section{Radu V Stan}

Dartmouth College Geisel School of Medicine

John Dessaint

Dartmouth-Hitchcock Medical Center

\section{Daniel S Aridgides}

Dartmouth-Hitchcock Medical Center

Diane L Mellinger

Dartmouth-Hitchcock Medical Center

Haley F Hazlett

Dartmouth College Geisel School of Medicine

Xiaoying Liu

Dartmouth-Hitchcock Medical Center

Alix Ashare

Dartmouth-Hitchcock Medical Center

\section{Research}

Keywords: Lung Macrophage, Vaping, Cytosolic inclusions

Posted Date: August 4th, 2020

DOI: https://doi.org/10.21203/rs.3.rs-51740/v1

License: (c) (i) This work is licensed under a Creative Commons Attribution 4.0 International License. Read Full License 


\section{Abstract}

Background: The effects of habitual vaping on lung health are currently unknown. Acute ecigarette/vaping product use-associated lung injury (EVALI) has been highlighted recently in numerous cases across the U.S. Several EVALI case reports highlight alterations in lung macrophages, justifying investigation of this key immune sentinel of the lung in habitual vape device users.

Methods: Histologic (Oil Red O stain) and transmission electron microscopy were used to evaluate BAL macrophages obtained from a habitual and asymptomatic 25 -year-old female vaper.

Results: Oil Red O stain evaluation of BAL macrophages demonstrate a prevalence of cells with high lipid accumulation in multiple, discrete cytoplasmic foci. At ultrastructural level, this translated in membranebound compartments filled with osmiophilic material of various densities segregated along curved phase separation lines reminiscent of suspensions of immiscible fluids.

Conclusions: This ultrastructural pattern is unlike any other previously reported in aspiration syndromes and may represent a defining diagnostic feature of vapers.

\section{Background}

The electronic nicotine delivery system (ENDS), entered the US market in 2007 and rapidly gained popularity among both tobacco smokers and non-smokers (1-4). E-juice, the liquid medium used in ENDS devices, contains one or more of the following chemicals: nicotine, THC, propylene glycol (PG) and vegetable glycerin (VG). In addition, polyethylene glycol, vitamin E acetate, or triglycerides are often added to e-juice to impact its viscosity (5). Vaping has short-term detrimental health effects on the lungs in certain individuals, a condition known as e-cigarette/vaping product use-associated lung injury (EVALI), and will likely have chronic effects that will not fully be realized for decades. Numerous EVALI case reports highlight alterations in lung macrophages (LMs) and suggest that the innate immune system may be affected in vape users(6-8). However, there are presently no studies in habitual vapers to examine/characterize pulmonary innate immune cells. We present a novel macrophage phenotype observed in BAL-derived LMs isolated from an asymptomatic habitual vaper.

\section{Case Presentation}

We present a case of an asymptomatic 25-year-old female frequent vape user with no underlying respiratory disease. The subject reported that she has been vaping for approximately 3.5 years with a daily consumption of 2 milliliters of e-juice including use of both nicotine-based and THC-containing products. The device she currently uses is SMOK brand, but the subject has also used JUUL brand. This subject is a previous cigarette smoker (3 years duration).

\section{Methods}


This study was approved by the Dartmouth-Hitchcock Institutional Review Board (\#22781). Subject underwent flexible bronchoscopy as previously described (9). Bronchoalveolar lavage (BAL) fluid was obtained from tertiary airways via instillation of $20 \mathrm{ml}$ of sterile saline followed by $10 \mathrm{ml}$ of air and repeated for a total of 5 times per airway. LMs were isolated as previously described $(9,10)$. Cells were stained with Oil Red O (ORO) to assess lipid accumulation (11). Cellular ultrastructure was examined by transmission electron microscopy (TEM). Cells prepared for TEM from a healthy non-smoking 27-year-old male were used for ultrastructural comparison.

\section{Results}

A heterogeneous distribution of ORO stain was seen across the LM cell population (Figure 1A). Some cells showed high accumulation of ORO staining droplets (arrow) while other cells showed little to no staining (arrowhead). Cells show ORO positive cytoplasmic droplets of varying size and varying subcellular localization (Figure 1B). We calculated a lipid-laden macrophage index (LLMI) from this cell prep via methods of Colombo and Halberg(11) of 172.5 (SD \pm 7.90$)$. Previous studies $(12,13)$ report LLMI < 50 from healthy subjects.

To gain insight into the nature of these lipid accumulations, LM ultrastructure was examined by TEM (Figure 2). LMs from both a healthy non-smoking subject (Panel A) and the habitual vaper (Panels B-F) were approximately $25-30 \mathrm{Im}$ in diameter. In the vaper LMs, ultrastructural analysis demonstrates heterogenous osmiophilic material accumulation in membrane bound intracellular compartments. We refer to this structure as a vaping-associated inclusion body (VIB). The abundance of VIBs is heterogeneous across the LM population and range in size from $500 \mathrm{~nm}$ to nearly $10 \square \mathrm{m}$ in diameter. Variable density inclusions were not observed in LMs of the non-smoking subject.

\section{Discussion}

The long-term effects of habitual vaping on pulmonary immune cells is unknown. Macrophages are among the first cells in the lung to respond to inhaled particles or pathogens. To explore possible phenotype alterations to LMs with vaping, we examine the cytology and ultrastructure of LMs from BAL fluid of a habitual vaping device user. We have identified a novel cytosolic accumulation of heterogeneous electron density which we refer to as a VIB, a vaping-associated inclusion body.

Historically, cytology-based studies utilizing Oil Red 0 staining have been used to demonstrate the presence of lipid-laden macrophages in the lung $(7,11,14,15)$ There are limited ultrastructural studies showing lipid-like cytosolic inclusions in lung macrophages from BAL fluid. M. tuberculosis infection studies $(16,17)$, mineral oil-exposure related $(18,19)$ or petroleum aspiration(20) reports come closest to describing similar inclusions, nonetheless these structures are clearly not the heterogeneous structures we have observed here in the vaping subject.

\section{Conclusion}


This is the first report describing lung macrophage ultrastructure associated with habitual vape product use. Most notably, we observed a novel pattern of cytosolic accumulation within membrane bound compartments with heterogenous electron density. This pattern does not resemble any pattern published in the prior literature, including vaping and aspiration pneumonia. One hypothesis of the likely composition of VIBs is that they are the vaporized derivatives of PG/VG in the e-juice. Future lipidomics analysis as well as organelle marker studies of LMs from habitual vapers may shed light on this question.

\section{Abbreviations}

LM: Lung Macrophage; BAL: Bronchoalveolar lavage; ENDS: electronic nicotine delivery system; EVALl: ecigarette/vaping, product use-associated lung injury; VIB: vaping-associated inclusion body; ORO: Oil Red O; PG/VG: propylene glycol/ vegetable glycerin

\section{Declarations}

This study was approved by the Dartmouth-Hitchcock Institutional Review Board (\#22781). The subject provided written consent for participation and publication of results (available upon request). All data is available from the corresponding author upon request. All authors state that there are no conflicts of interest (financial nor personal) associated with this work. "Disclosures: None declared."

Funding: This work is supported by the NIH R01HL122372 (to A.A.) and NIH R01CA216265 (to B.C.C), CFF ARIDGI19B0 (to D.S.A.) and R01GM120592 (to R.V.S.). Subject enrollment and clinical sample collection were achieved with the assistance of the Translational Research Core at Dartmouth, which is jointly funded by the NIH (P30DK117469 to Dean Madden) and CFF (STANT019R0 to Bruce Stanton). "The funders (Madden and Stanton) had no role in study design, data collection and analysis, decision to publish, or preparation of the manuscript."

Authors' contributions: DAA and AA conceived the study. DAA, RVS, JAD, DSA, DLM, HFH and XL assisted in sample collection and analyzed the data. DAA wrote the manuscript. All authors edited and approved final manuscript.

\section{References}

1. Madison MC, Landers CT, Gu BH, Chang CY, Tung HY, You R, et al. Electronic cigarettes disrupt lung lipid homeostasis and innate immunity independent of nicotine. J Clin Invest. 2019;129(10):4290304.

2. Regan AK, Promoff G, Dube SR, Arrazola R. Electronic nicotine delivery systems: adult use and awareness of the 'e-cigarette' in the USA. Tob Control. 2013;22(1):19-23.

3. Gentzke AS, Creamer M, Cullen KA, Ambrose BK, Willis G, Jamal A, et al. Vital Signs: Tobacco Product Use Among Middle and High School Students - United States, 2011-2018. MMWR Morb Mortal Wkly 
Rep. 2019;68(6):157-64.

4. Mirbolouk M, Derakhshan A, Charkhchi P, Guity K, Azizi F, Hadaegh F. Incidence and predictors of early adulthood pre-diabetes/type 2 diabetes, among Iranian adolescents: the Tehran Lipid and Glucose Study. Pediatr Diabetes. 2016;17(8):608-16.

5. Gutsche J, Pasternak R, Campbell D, Schili JL, Boyle PJ, Tilney P. A 19-Year-Old Man With VapingAssociated Lung Injury. Air Med J. 2020;39(1):6-8.

6. Butt YM, Smith ML, Tazelaar HD, Vaszar LT, Swanson KL, Cecchini MJ, et al. Pathology of VapingAssociated Lung Injury. N Engl J Med. 2019;381(18):1780-1.

7. Guerrini V, Panettieri RA, Jr., Gennaro ML. Lipid-laden macrophages as biomarkers of vapingassociated lung injury. Lancet Respir Med. 2020;8(2):e6.

8. Maddock SD, Cirulis MM, Callahan SJ, Keenan LM, Pirozzi CS, Raman SM, et al. Pulmonary LipidLaden Macrophages and Vaping. N Engl J Med. 2019;381(15):1488-9.

9. Bessich JL, Nymon AB, Moulton LA, Dorman D, Ashare A. Low levels of insulin-like growth factor-1 contribute to alveolar macrophage dysfunction in cystic fibrosis. J Immunol. 2013;191(1):378-85.

10. Chen Y, Armstrong DA, Salas LA, Hazlett HF, Nymon AB, Dessaint JA, et al. Genome-wide DNA methylation profiling shows a distinct epigenetic signature associated with lung macrophages in cystic fibrosis. Clin Epigenetics. 2018;10(1):152.

11. Colombo JL, Hallberg TK. Recurrent aspiration in children: lipid-laden alveolar macrophage quantitation. Pediatr Pulmonol. 1987;3(2):86-9.

12. Gibeon D, Zhu J, Sogbesan A, Banya W, Rossios C, Saito J, et al. Lipid-laden bronchoalveolar macrophages in asthma and chronic cough. Respir Med. 2014;108(1):71-7.

13. Knauer-Fischer S, Ratjen F. Lipid-laden macrophages in bronchoalveolar lavage fluid as a marker for pulmonary aspiration. Pediatr Pulmonol. 1999;27(6):419-22.

14. Layden JE, Ghinai I, Pray I, Kimball A, Layer M, Tenforde MW, et al. Pulmonary Illness Related to ECigarette Use in Illinois and Wisconsin - Final Report. N Engl J Med. 2020;382(10):903-16.

15. Ocampo-Gonzalez FA, Park JW. Cytologic features of vaping-induced lung injury: A case report. Diagn Cytopathol. 2020;48(2):174-6.

16. Armstrong JA, Hart PD. Response of cultured macrophages to Mycobacterium tuberculosis, with observations on fusion of lysosomes with phagosomes. J Exp Med. 1971;134(3 Pt 1):713-40.

17. Borelli V, Vita F, Soranzo MR, Banfi E, Zabucchi G. Ultrastructure of the interaction between mycobacterium tuberculosis- H37Rv-containing phagosomes and the lysosomal compartment in human alveolar macrophages. Exp Mol Pathol. 2002;73(2):128-34.

18. Ohwada A, Yoshioka Y, Shimanuki Y, Mitani K, Kumasaka T, Dambara T, et al. Exogenous lipoid pneumonia following ingestion of liquid paraffin. Intern Med. 2002;41(6):483-6.

19. Osman GA, Ricci A, Terzo F, Falasca C, Giovagnoli MR, Bruno P, et al. Exogenous lipoid pneumonia induced by nasal decongestant. Clin Respir J. 2018;12(2):524-31. 
20. Burkhardt O, Merker HJ, Shakibaei M, Lode H. Electron microscopic findings in BAL of a fire-eater after petroleum aspiration. Chest. 2003;124(1):398-400.

\section{Figures}
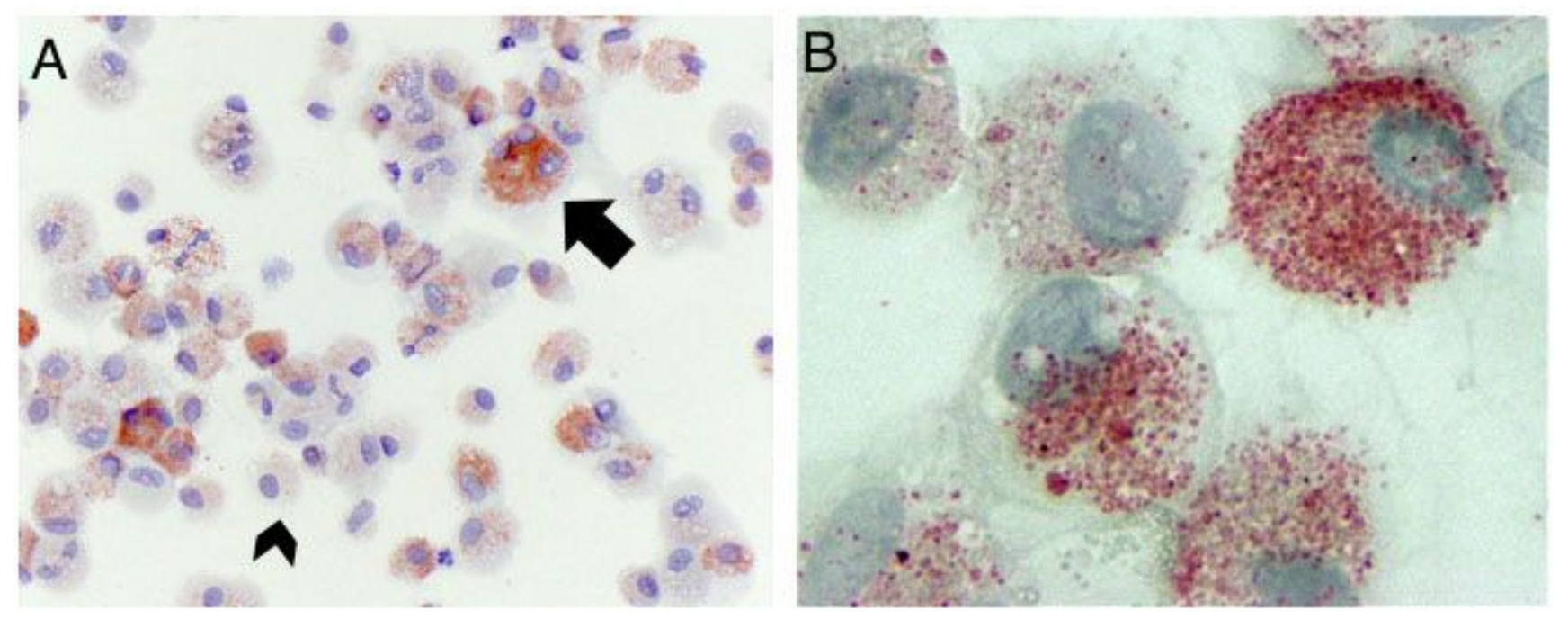

\section{Figure 1}

Lung Macrophages from an Asymptomatic Vaper Stain Positive for Oil Red O. Primary human lung macrophages (LMs) were prepared via cytospin and stained for lipid content with Oil Red O (ORO). Heterogeneity of ORO staining across the cell population (Panel A) (Magnification $400 \mathrm{x}$ ). Higher magnification of LMs (1000 x, Panel B) further illustrates both the ORO staining heterogeneity between cells and the size of the cytoplasmic organelles staining ORO positive. 


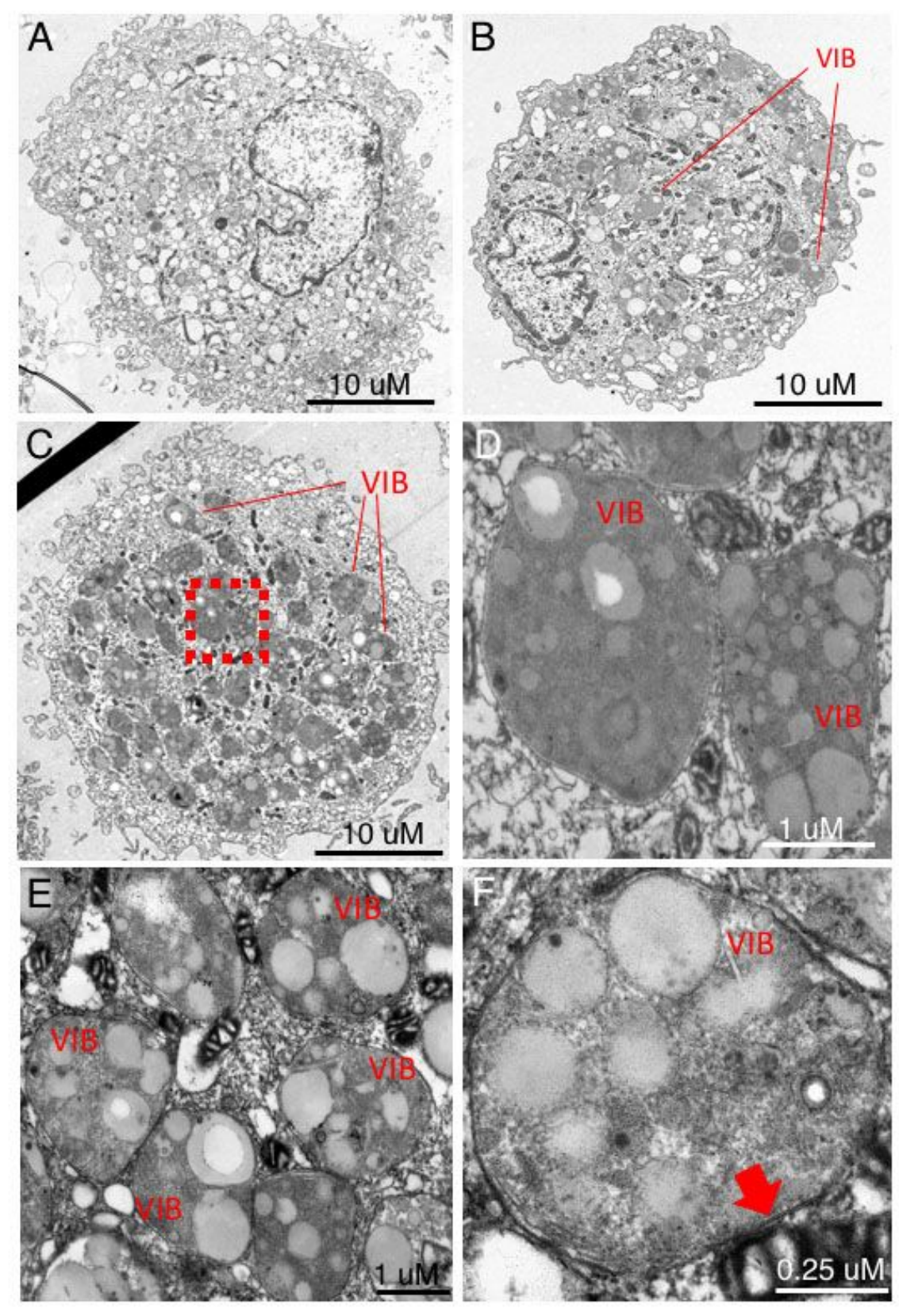

\section{Figure 2}

Human Lung Macrophages from Asymptomatic Vaper Contain Novel Inclusion Bodies. Transmission electron micrographs of primary lung macrophages (LM) collected by bronchoalveolar lavage from a non-smoker (A) and a frequent vaper (B-F). Common features in LMs from both subjects were numerous mitochondria, translucent vacuoles and cells approximately 25-30 uM in diameter. A) Representative image of a LM from the non-smoker. B) Vaper LM demonstrating a novel ultrastructural feature we refer 
to as a vaping-associated inclusion body (VIB). C-D) Vaper LM with a high abundance of inclusion bodies (red box outlines the region selected for higher magnification imaging in D). D-F) Higher magnification of the VIB ultrastructure reveals multiple regions of varying electron density within the organelle. Panel $F$ shows a lipid bilayer surrounding the inclusion body (red arrow).

\section{Supplementary Files}

This is a list of supplementary files associated with this preprint. Click to download.

- 200730RespiratoryResearchCOVERLetter.docx 